\title{
Evaluación de impactos de una intervención preventiva de drogas y alcohol: El caso del programa A Tiempo en la comuna de San Pedro de la Paz
}

\author{
Carolina Paz Núñez Vera** \\ José Manuel Merino Escobar ${ }^{* * *}$
}

RESUMEN

\begin{abstract}
Existe un creciente interés en implementar evaluaciones orientadas a determinar impactos generados por programas sociales. Este trabajo presenta el efecto del Programa de Prevención de Alcohol y Drogas "A Tiempo" sobre cuatro dimensiones de riesgo en 44 estudiantes de la comuna de San Pedro de la Paz, Región del Biobío, durante el año 2012. Los datos fueron obtenidos a partir de lo realizado por el equipo del Servicio Nacional para la Prevención y Rehabilitación del Consumo de Drogas y Alcohol (SENDA), lo que permitió realizar un esquema pre-post test sin grupo contraste. La prueba utilizada fue T-Student para muestras relacionadas. Los resultados nos permitieron establecer que sólo existen disminuciones significativas en las dimensiones de riesgos individual y familiar, aunque se advierten cambios en dimensión pares y de consumo que deben ser mayormente observadas.
\end{abstract}

Palabras clave: factores de riesgo, drogas, alcohol, contexto escolar, evaluación, modelo pre-post.

Avaliação de impactos de uma intervenção preventiva de drogas e álcool: O caso do programa Em Tempo na comuna de São Pedro da Paz

RESUMO

Existe um interesse crescente em implementar avaliações orientadas a determinar impactos gerados por programas sociais. Este trabalho apresenta o efeito d Programa de Prevenção de Álcool e Drogas "Em

Artículo recibido: 14/05/2014. Artículo aprobado: 20/08/2014. Versión final: 30/09/2014.

* Chilena. Socióloga. Magíster en Trabajo Social y Políticas Sociales, Universidad de Concepción. Jefa Departamento de Educación, I. Municipalidad de Lota, Chile. Correo electrónico: caronunez@udec.cl

..* Chileno. Sociólogo, Doctor en Sociología, Profesor titular en Universidad de Concepción, Chile. Correo electrónico: jmerino@udec.cl 
Tempo" sobre quatro dimensões de risco em 44 estudantes da comuna de São Pedro da Paz, Região do Biobío, durante o ano 2012. Os dados foram obtidos a partir do realizado pela equipe de Serviço Nacional de Prevenção e Reabilitação de Drogas e Álcool ( SENDA, o que permitiu realizar um esquema pré-post test sem grupo de contraste. O teste utilizado foi o T-Student para mostras relacionadas. Os resultados permitiram-nos estabelecer que somente existem diminuições significativas nas dimensões de risco "individual" e "familiar", ainda de que se advertem alterações na dimensão "pares" e "de consumo" que devem ser maiormente observadas.

Palavras-chave: fatores de risco, drogas, álcool, contexto escolar, avaliação, modelo de pre-post.

\title{
Assessment of impacts of a drug and alcohol preventive intervention program: The case of A tiempo in San Pedro de la Paz Municipality
}

\author{
ABSTRACT
}

There is a growing interest for implementing assessments aimed at determining the impacts of social programs. This paper presents the outcome of the Alcohol and Drug Abuse Prevention Program "A tiempo" (On time) on four risk dimensions in 44 students in the municipality of San Pedro de la Paz, Biobío Region, in 2012. Data were collected from the work of National Service for Prevention and Rehabilitation of Drug and Alcohol (SENDA) working team, which allowed designing a pre-post test scheme without a contrast group. The test used was T-Student for related samples. The results allowed us to establish that there are only significant decreases in the dimensions of "individual" and "family" risk, although changes are noted in the dimensions "peers" and "consumer", which have to be more deeply observed.

Keywords: risk factors, drugs, alcohol, school context, evaluation, pre-post model.

\section{Antecedentes}

La temática de las drogas, considerando sus diferentes procesos ${ }^{1}$ y a raíz de sus nuevas expresiones ${ }^{2}$, se ha vuelto parte constante de las agendas de los Estados y de la discusión social. A través de lo informado por la Oficina de las Naciones Unidas en Drogas y Crimen, UNODC (2012), el consumo de drogas ilícitas en el mundo se caracteriza por concentrarse en la población juvenil

Resumidas en: producción, tráfico, consumo.

Ingreso de nuevas sustancias al mercado de las sustancias lícitas e ilícitas; nuevas formas de "promoción" de éstas, entre otras expresiones actuales. 
del sector urbano y por la tendencia creciente al uso de sustancias psicoactivas, en especial en países en desarrollo como es el caso de Chile. Otra tendencia creciente es que en población joven no existe mayor disparidad de consumo entre géneros, a diferencia de la población adulta que presenta mayor consumo masculino en casi todos los países del mundo. Adicionalmente, en Chile existe preocupación por la tendencia al incremento en el segmento escolar de la percepción de presencia de drogas en sus entornos más próximos, además de un incremento en la facilidad de su obtención, que se traduce en una amplificación del riesgo de consumo en niños, niñas y adolescentes, quienes dado su ciclo de vida cuentan con mayores espacios de proximidad con distintos actores sociales, en especial con el grupo de pares, y presentan una serie de cambios a nivel de la personalidad que impactan en su construcción personal, y que finalmente ajusta sus futuros comportamientos sociales (Ortiz et al., 2005).

Según los Estudios Nacionales de Drogas en Población Escolar en Chile (SENDA, 2013), el consumo de estupefacientes es frecuente entre la población escolar, siendo la marihuana la sustancia ilícita de mayor utilización: más de 10\% de la población entre 16 y 18 años la consume. Además se ha observado que la prevalencia de consumo ${ }^{3}$ de alcohol desde la adolescencia temprana va en aumento: alrededor de un cuarto de los y las escolares entre los 13 y 15 años, y poco menos de la mitad de los de entre 16 y 18 años consumen alcohol mínimo una vez al mes. Para el consumo de cigarrillos, se observa que desde el año 1994 ha ido decreciendo el porcentaje de adolescentes que declara consumir tabaco, siendo el descenso más importante el ocurrido entre el año $2010(11,1 \%)$ y $2012(6,4 \%)$.

Autores como Trudel \& Puentes Neuman (2000) agregan que la condición de estar en riesgo se define por la relación entre factores externos y vulnerabilidades individuales que al entrar en interacción incrementan las probabilidades de desarrollar problemas emocionales, conductuales o de salud; y por tanto de presentar

Prevalencia como medida para determinar magnitud de consumo: para drogas ilícitas comunes como marihuana, pasta base y cocaína es una vez en el último año; mientras que para las lícitas como alcohol y cigarrillo, si ha consumido durante los últimos 30 y 20 días respectivamente. 
una conducta de riesgo ${ }^{4}$. En este sentido cabe destacar los resultados de estudios del área de la epidemiología que dan cuenta de una relación entre el uso abusivo de drogas y conductas infractoras de ley en adolescentes (Villatoro et al., 2002), constituyéndose ambas en conductas de riesgo que tienden a darse en conjunto ${ }^{5} \mathrm{y}$ relacionarse en dos sentidos: tanto cuando el consumo constituye un delito en sí o éste se comete para financiar una adicción, como en sentido inverso, cuando las personas ingieren alcohol o drogas para atreverse a cometer un delito (Hurtado, 2005).

Donaldson (2009) encontró que las personas jóvenes que abusan del alcohol podrían desarrollar déficit en sus desarrollos cerebrales estructurales y funcionales comparados con quienes no lo hacen, y que pueden presentar efectos adversos también en huesos y en su desarrollo endocrino. Jit y colaboradores (2009) y ASH (2014) han resuelto que los y las adolescentes que presentan consumo temprano de cigarrillos están asociados con altos niveles de dependencia, alto consumo, menor chance de terminar la adicción y una más alta mortalidad; inclusive algunos han llegado a determinar que la probabilidad de dependencia al alcohol puede ser reducida un 10\% por cada año de retraso en el inicio del uso de alcohol, lo que muestra la relevancia de generar campañas preventivas del consumo y promoción de vida sana (Grant et al., 2001). De esta forma se abre una serie de desafíos para los Estados, pero también para quienes se encuentran en el área de la investigación y del diseño de políticas dirigidas a los niños, niñas y adolescentes. Poniendo acento justamente en esto es que se asienta la Prevención del consumo de drogas y alcohol en el marco de políticas para hacer frente al consumo, entendida como una diversidad de estrategias dinámicas que tienen como objetivo principal eliminar o reducir los problemas derivados de la indebida utilización de sustancias antes de que comiencen (CSAP, 1997); O, como define Monreal y asociados (2009), serie de acciones que permitan evitar o postergar el consumo de drogas en una población determinada. Así, y en vista a desarrollar programas eficaces se ha priorizado por iniciativas destinadas a

De esta forma se genera una distinción entre factor de riesgo y conductas de riesgo, las que se definen como el resultado que tiene la acción de los factores en un individuo (Hein, 2004).

5 Aunque los estudios revisados para esta investigación muestran una relación entre consumo de drogas y actos delictivos, no se ha encontrado consenso en determinar causalidad entre ambos fenómenos. 
prevenir el consumo, más que por medidas paliativas vinculadas con la asistencia sanitaria.

Se han formulado una serie de teorías respecto al consumo de drogas que tienen como base Teorías del Aprendizaje Social, Conductas problemáticas, Autoestima, Responsabilidad del Grupo de Pares y las Comunicaciones Persuasivas (Botvin, 2001). En la actualidad el diseño de acciones dirigidas a afrontar el consumo, uso y abuso de drogas han ido haciéndose mucho más específicas dado el aumento de la diversidad de sustancias, sujetos y contextos; considerando la multiplicidad de elementos intervinientes sobre el sujeto consumidor ${ }^{6}$ y restándole así una carga estigmatizadora al evento del consumo. Los enfoques utilizados han transitado desde la concepción del modelo sanitario hasta un enfoque de factores psicológicos y sociales de competencia, resiliencia y el de factores protectores y de riesgo. Así por tanto, responden a lineamientos teóricos formulados para la atención del problema y para la promoción del cambio (NIDA, 2006; OPS, 2011). A su vez, estos enfoques han propiciado diferentes estrategias preventivas que se definen como el conjunto de actividades, actuaciones y/o servicios que constituyen la intervención. La literatura señala distintas clasificaciones de niveles preventivos, entre los que destacan: inespecífica/específica, oferta/demanda, segmento poblacional, primaria, secundaria o terciaria (Alvira, 1999), y la prevención estratificada propuesta inicialmente en 1987 por Gordon (citado en Becoña, 2002).

En esta investigación tuvimos como objeto responder a la pregunta: ¿el programa A Tiempo genera un cambio significativo en la presencia de factores de riesgo y consumo de drogas y alcohol en los y las estudiantes de la comuna de San Pedro de La Paz que participaron durante el año 2012? Sólo la evaluación de los efectos de los programas sociales permite analizar en qué áreas ocurren cambios en el fenómeno sobre el que se actúa y con esto, reflexionar en torno a mejoras de las políticas públicas orientadas a hacerle frente (Peroni, 2012). Tal como señalan Cohen y sus colaboradores (2000), la evaluación meticulosa de las intervenciones debe formar parte integral del proceso de toma de decisiones en materia de políticas sociales.

Hawkins, Catalano \& Miller, 1992; Newcomb \& Bentler, 1989, citados en Botvin, 2000 


\section{Programa A Tiempo en la comuna de San Pedro de la Paz, Región del Biobío, año 2012}

El Programa A Tiempo está diseñado como una intervención temprana del consumo de drogas y alcohol que implica la detección pre$\mathrm{coz}$ de factores de riesgo en adolescentes escolarizados. Trabaja en establecimientos educacionales dada la facilidad para identificar a la población objetivo del programa, la sistematicidad en el trabajo de los y las estudiantes en las sesiones programadas y la capacidad para trabajar de modo más integral aprovechando las otras instancias presentes en los establecimientos educacionales; abordando así condiciones que podrían actuar como factores de riesgo del consumo tanto a nivel individual como social: en la convivencia que se da en la escuela y en los posibles espacios de participación que ésta promueva y que impacten positivamente en una cultura de autocuidado ${ }^{7}$. El objetivo general del programa es aumentar factores protectores y disminuir factores de riesgo en estudiantes de establecimientos educacionales focalizados para prevenir el consumo de drogas y alcohol, mediante la implementación de estrategias integrales de trabajo. La intervención es en 6 sesiones individuales con temas pauteados, vinculados con habilidades sociales, capacidad de resolución de conflicto, actitudes y valores; 6 sesiones de trabajo grupal donde se trabajan temáticas de inclusión/exclusión, bullying, sentido de pertenencia y riesgos, actividades importantes de acuerdo al ciclo vital, y 3 sesiones con asistencia familiar para tratar temas vinculados a la cohesión familiar, estilos parentales, estresores de la familia como pobreza, vulnerabilidad, entre otros temas. El Programa está presente a nivel nacional y es ejecutado por equipos en cada municipio, quienes realizan la focalización a través de la selección de Establecimientos Educacionales siguiendo criterios de vulnerabilidad, tales como que percibieran Subvención Escolar Preferencial (SEP) o tuvieran un 70\% o más en el Índice de Vulnerabilidad Escolar del Sistema Nacional de Asignación con Equidad (IVE SINAE). La implementación y ejecución del Programa A Tiempo en San Pedro de la Paz estuvo a cargo del equipo compuesto por un coordinador y una dupla psicosocial perteneciente al SENDA de esa comuna,

Faggiano y su equipo (2007) destacan que se debe fortalecer los espacios de convivencia como un factor protector frente a conductas de riesgo en población adolescente. 
la cual se ubica al sur de la Provincia de Concepción en la Región del Biobío, Chile.

\section{Metodología}

Fue de carácter cuantitativo longitudinal, enmarcado dentro de los diseños cuasi-experimentales, con mediciones pre y postintervención sin grupo control. El muestreo fue de tipo no probabilístico con selección intencionada de casos en 4 Establecimientos Educacionales que cumplían las condiciones de focalización requeridas por la programación nacional. Estos fueron el Colegio Michaihue, Colegio Luterano Piececitos, Colegio Nuevos Horizontes y Escuela Sargento Candelaria Pérez. La unidad de análisis correspondió a estudiantes focalizados por el Programa que voluntariamente accedieron a participar en la intervención (56 casos); sin embargo, la muestra quedó constituida por 44 casos, ya que sólo se consideraron a quienes asistieron al menos a una de las sesiones del Programa y que cuentan con medición de factores de riesgo posterior a la intervención, lo que dio 22 hombres y 22 mujeres, con edades que fluctuaron entre los 11 y los 17 años. La técnica utilizada fue la de recolección de datos secundarios proporcionados por el SENDA de la comuna de San Pedro de la Paz, quienes originalmente utilizaron un cuestionario de autoaplicación ${ }^{8}$ para determinar la presencia de factores de riesgo y una entrevista semiestructurada ${ }^{9}$. La variable dependiente corresponde a la presencia de Factores de Riesgo y de Consumo de Drogas y/o Alcohol presentes en los y las adolescentes participantes del Programa; factores entendidos como ciertas condiciones que hacen que la conducta riesgosa sea más probable de aparecer, y se agruparon en 5 dimensiones: Familia, se evalúa con dos escalas Actitudes de los padres frente al consumo, y Nivel de Involucramiento o Manejo que éstos tienen sobre la conducta de sus hijos/as adolescentes, con un puntaje total de 34 puntos; Individual, que tiene relación a cómo los adolescentes perciben e interiorizan el riesgo latente por consumir drogas y/o al-

\footnotetext{
La elaboración estuvo a cargo de una Mesa Técnica constituida por profesionales del SENDA, a través de un meta análisis y un análisis factorial y de confiabilidad de la experiencia de 24 escalas; quedando finalmente el instrumento constituido por 6 escalas de factores de riesgo en formato Likert, representativas de 4 dimensiones que se componen de entre 3 a 7 ítems cada una, donde a mayor puntaje, mayor el nivel de riesgo presente (SENDA, 2012)

9 Consta de una serie de preguntas y sugerencias para el abordaje mismo de la entrevista.
} 
cohol, y la actitud que adoptan frente a las drogas, y que suman como máximo 22 puntos; Grupo de Pares estuvo compuesta por la escala Recompensas o gratificaciones de los amigos por consumo de drogas y corresponde al comportamiento que supone de su grupo de pares frente a un consumo de sustancias, con un total máximo de 9 puntos; y Conductas de riesgo que está compuesta por una escala denominada Consumo y que mide las experiencias de consumo del o la adolescente al momento de la medición, con un puntaje máximo de 13 puntos de riesgo; y una Dimensión Total u Overall que corresponde a la suma de los puntajes en las 4 dimensiones, con un puntaje máximo de riesgo de 78 puntos. La suma de puntajes obtenidos para cada caso en cada dimensión permitió establecer la presencia de factores de riesgo que por sí solos no aumentan la probabilidad de consumo sino que actúan en conjunto mediante su acumulación. La variable predictora corresponde a la participación en el Programa A Tiempo que tiene como objetivo principal prevenir el consumo en contextos escolares pertenecientes a sectores vulnerables, y que contemplaba diferentes actividades tales como consejerías individuales, actividades grupales, orientación o asesoría familiar y vinculación con red de apoyo*, de mínimo 15 sesiones para el trabajo. Es importante la evaluación de los factores de riesgo a los que puede estar expuesta una persona, en el entendido de estimar la probabilidad que ocurra determinado consumo, sin que establezca necesariamente relaciones causa-efecto.

Los datos, facilitados por el equipo del SENDA de la comuna de San Pedro de la Paz, fueron recolectados en dos momentos: marzo y abril para el pre test y noviembre de 2012 para el post test en los establecimientos educacionales suscritos. Posteriormente codificamos y tabulamos en Microsoft Excel para finalmente analizarlos con el paquete IBM SPSS Statistics versión 20. Para determinar efectos de la intervención se consideró hacerlo en función de posibles disminuciones significativas de la presencia de factores de riesgo, adoptándose para el análisis de los datos una lógica paramétrica a través de la aplicación de la prueba T-Student para muestras pareadas; lo que permite determinar diferencias significativas entre las medias de los puntajes obtenidos al ingreso y al egreso del Programa que podrían atribuirse a la experiencia de participación en la intervención. El nivel de error máximo utilizado fue de 0.05 . 


\section{Resultados}

El total de estudiantes presentó entre 11 y 17 años de edad y un valor modal de 13 y 14 años. Esto toma relevancia al considerar que el ciclo de vida al que apunta el programa direcciona y rige la organización de las actividades, los énfasis y la definición de los temas a tratar considerando las particularidades de la etapa: importancia del grupo de pares, importancia de la familia, inicio de la vida sexual, definiciones de género, adaptaciones al medio social, entre otros factores. Respecto a la composición por nivel educacional, se observó una concentración en nivel básico (79,5\%), que junto con la variable edad del estudiante permite ver un leve rezago educativo ${ }^{10}$ y que correspondería a un factor de riesgo escolar considerando que puede traducirse en abandono del sistema (INEE, 2010) y en un factor de riesgo de abuso de sustancias (Petterson et al., 1992, citado en Becoña, 2000). La adherencia al Programa también resulta ser algo importante a presentar ya que como postulan Gottfredson $\mathcal{E}$ Wilson (2003), tanto el método, la duración y los tiempos de intervención son variables que moderan los resultados de un trabajo psicosocial ${ }^{11}$. Se observó que la asistencia total a la intervención (asistencia del adolescente a las sesiones individuales, grupales y con la familia) promedió 56,4\%, lo que implica éxito, ya que el Programa A Tiempo espera que al menos los y las jóvenes asistan al 50\% de las sesiones programadas.

La Dimensión Total de Riesgos presentes u Overall se considera como el resultado total de presencia de factores de riesgo de los y las adolescentes participantes en el Programa. Por esto se realizó un análisis de correlación mediante la prueba de Pearson para establecer covariación entre los puntajes globales antes y después que podría asociarse a la influencia del programa; indicando los resultados con un nivel de error de 0.001 una correlación positiva de fuerza mediana $(0,503)$. La comprobación de significación de esta variación entre los puntajes totales antes $(27,02)$ y después de la intervención $(20,25)$ realizada con T-student para muestras relacionadas, mostró una disminución de 6,77 puntos promedio en la presencia de riesgos totales; lo que en virtud de su valor de

10 Entendido como no contar con el nivel que de acuerdo a su edad está establecido y acorde a las disposiciones del Ministerio de Educación de Chile.

11 Encontrándose evidencia por ejemplo, que asocia mejores efectos a intervenciones con mayor cantidad de sesiones y a sesiones que tienen entre 11 y 30 horas respecto a las que tienen 10 o menos (Gottfredson, et al., 2003) 
significación ( $\mathrm{p}=0.001$ ) permite aseverar que sí difieren de manera estadísticamente significativa, y por tanto hay una baja real de la presencia de factores de riesgo en la evaluación post intervención.

Posteriormente se realizaron los análisis de correlaciones para las 4 dimensiones que miden presencia de riesgos en los y las adolescentes intervenidos: Familiar, Individual, Grupo de Pares y Conductas de riesgo, observándose en todas diferencias entre los puntajes de factores de riesgo presentes en los y las adolescentes antes de someterse a la intervención y al finalizar el Programa. La Tabla siguiente muestra los valores promedios para cada dimensión después y antes de la intervención del A Tiempo y la relación a través del coeficiente de Pearson:

Tabla 1. Estadísticos y correlaciones pre-post por dimensiones de riesgo

\begin{tabular}{|l|l|l|l|l|}
\hline Dimensiones & pre & post & r & $p$ \\
\hline Familia & 12.39 & 9.07 & 0.557 & 0.000 \\
\hline Individual & 9.59 & 6.95 & 0.383 & 0.010 \\
\hline Pares & 3.75 & 2.98 & 0.350 & 0.020 \\
\hline Conductas de riesgo & 1.32 & 1.25 & 0.350 & 0.020 \\
\hline
\end{tabular}

Fuente: Análisis de Datos Proyecto Evaluación A Tiempo 2012.

Es así que todos los coeficientes de correlación para las dimensiones de riesgo que constituyen el instrumento con el que se tamiza la presencia de riesgos en los y las estudiantes que participan en el programa muestran relaciones de intensidad baja a media, con niveles de significación estadística que apoyan la relación existente entre ambos momentos $(p<0.05)$. Al igual que en el caso anterior, luego de este análisis, se sometieron cada una de las dimensiones a la prueba de comparación de medias T-student para muestras relacionadas, la que entregó los siguientes resultados:

Tabla 2. Prueba T-Student para muestras pareadas

\begin{tabular}{|l|l|l|l|l|l|}
\hline Dimensiones & & Dt & $\mathrm{t}$ & $\mathrm{gl}$ & $\mathrm{p}$ \\
\hline Familiar Post-Pre & -3.31 & 5.74 & -3.83 & 43 & 0.00 \\
\hline Individual Post-Pre & -2.63 & 6.56 & -2.66 & 43 & 0.01 \\
\hline Grupo de Pares Post-Pre & -0.77 & 2.93 & -1.74 & 43 & 0.08 \\
\hline $\begin{array}{l}\text { Conductas de Riesgo } \\
\text { Post-Pre }\end{array}$ & -0.06 & 2.49 & -0.18 & 43 & 0.85 \\
\hline
\end{tabular}

Fuente: Análisis de Datos Proyecto Evaluación A Tiempo 2012. 
De esta forma se determinó que tanto las dimensiones Familia e Individual presentaron variaciones significativas ( $p<0,01)$, mostrando que la dimensión familiar tuvo en promedio 3,31 puntos menos, en tanto que para la dimensión individual, los sujetos vieron reducida la presencia de riesgos en 2,63 puntos luego de participar en el programa. A pesar de que para las dimensiones Pares y Conductas de Riesgo el promedio de los factores de riesgos presentes entre la primera y la segunda medición también sufrió una reducción (menor a 1 punto en las dos dimensiones), sus niveles de error fueron superiores a lo aceptado. Esto permite concluir que de las 4 dimensiones que miden presencia de riesgos de consumo de alcohol y drogas, sólo en 2 dimensiones correspondientes a Familia e Individuos las diferencias encontradas son significativas y podrían atribuirse al paso de los y las adolescentes por el Programa. Luego de establecidos los resultados de cambios para el total de Dimensiones de Factores de Riesgo puede establecerse que es real la disminución de los factores de riesgo para los adolescentes y que podría atribuírsele al Programa y por tanto entenderse como efecto de la intervención.

\section{Conclusiones}

La investigación realizó un análisis de los cambios producidos en adolescentes luego de su participación en una intervención temprana dispuesta por el gobierno de Chile para la detección temprana y el trabajo preventivo del consumo de drogas y alcohol en adolescentes, denominada A Tiempo. La información con la que contaba el programa generó una evaluación con un diseño sin grupo control, por lo que no pueden establecerse relaciones de causalidad respecto a los efectos generados. A diferencia de un experimento social con asignación aleatoria, donde las unidades de análisis son asignadas mediante azar al grupo control y al tratamiento, en este tipo de investigación cuasi experimental donde los grupos están formados (o para nuestro caso donde no se contó con uno), no se puede estar seguro de que no existan diferencias preexistentes o provocadas por variables externas al programa, lo que para esta investigación se controló con una prueba robusta de efectos fijos como es el T student. Siguiendo esto es que se plantea como necesario asegurar que en el diseño mismo de los programas consideren realizar seguimiento, monitoreo o evaluación de resultados proyectados. 
Diversas investigaciones han demostrado la importancia de generar programas preventivos usando el contexto escolar (Thomas et al., 2006; Faggiano et al., 2007, ambos citados en James, 2011), y se ha demostrado que producen un impacto en las sustancias más comúnmente consumidas por los y las adolescentes, como son el tabaco, el alcohol y la marihuana (Foxcroft et al., 2011, en James, 2011). Por esto en particular nos propusimos evaluar si el programa cumple con su objetivo principal de aumentar los factores protectores y disminuir los factores de riesgo a población estudiantil de establecimientos vulnerables, tomando el caso de la comuna de San Pedro de la Paz, ejecutado el año 2012.

En referencia al principal objetivo de la investigación, que corresponde a determinar los efectos de la intervención del Programa en los y las adolescentes que participaron de él durante el año 2012, el análisis por Dimensión de Riesgo Total reunió el puntaje total que obtuvieron los adolescentes antes y después de participar en las actividades preventivas del programa y los analizó, logrando determinar que el riesgo promedio al que estuvo expuesta la muestra pre intervención fue de 27,02 y el resultante post intervención fue de 20,25 puntos de un máximo teórico de 78 puntos para la dimensión total. También, que esta diferencia entre ambos puntajes ( $-6,77$ puntos promedio), aunque es modesta tiene significación estadística, lo que se traduce en que esta disminución en la presencia de factores de riesgos a los que estuvieron expuestos los y las estudiantes terminando el programa puede ser atribuible a éste. Respecto al tamaño del efecto encontrado, aunque corresponde a una medida pequeña de cambio, y siguiendo lo que propone James (2011), por más modesto que éste sea, de igual forma es un resultado valioso, considerando los daños asociados al consumo temprano en adolescentes; y en esto radica la diferencia: al intervenir población adolescente cualquier efecto es relevante en términos de distanciar o aminorar el consumo de drogas y alcohol.

La participación en acciones preventivas como las diseñadas por el Programa con grupo de pares y el desarrollo de habilidades interpersonales como las trabajadas en las sesiones individuales, grupales y familiares, son respaldadas por diversas investigaciones como muy efectivas (Miller \& Rollnick, 1999, 2002; Velásquez et al., 2001, citado en SENDA, 2012a; Zweben \& Zuckoff, 2002). Los resultados de esta investigación también mostraron 
que las Dimensiones Grupo de Pares y Conductas de Riesgo, si bien presentan disminuciones en los puntajes de riesgo en el antes y después, sus niveles de error superan el 0.05. Uno de los argumentos que se esgrimen para esta falta de significación estadística de la diferencia es que en contraste de las dimensiones que resultaron significativas, el Grupo de Pares y la Conducta de riesgo reunían sólo 1 escala con 3 factores, lo que hacía decrecer la fortaleza de la medición, lo que no implica que la dimensiones en términos teóricos no deban considerarse cuando se establecen las dimensiones para medir riesgos. La detección temprana del consumo experimental o el uso de sustancias nocivas pero lícitas es justamente a lo que apuntan los programas preventivos con buen criterio (Becoña, 2000), ya que aunque no hay una linealidad segura, se ha demostrado que el consumo de tabaco y alcohol aumenta la probabilidad de consumir marihuana (Becoña, 2002) y el consumo de marihuana a su vez aumenta la probabilidad de experimentar con otras drogas sintéticas o semisintéticas. En referencia a lo mismo, es relevante destacar que se determinó que un porcentaje muy menor, alrededor de 12 casos, presentaban algún nivel de consumo tanto de cigarrillos como de alcohol, mientras que los consumos de marihuana y cocaína o pasta base estuvieron presentes en una pequeña porción de los y las estudiantes participantes del Programa; presentándose como particularidad que quienes declararon consumir marihuana incrementaron entre la primera y la segunda medición (de 6 a 11 casos). Específicamente para el caso de esta Dimensión pudo determinarse mediante entrevista con el profesional de la dupla psicosocial como informante clave, que lo declarado en el cuestionario no es reflejo de la situación de consumo de los y las estudiantes, la que era de mayor riesgo ${ }^{12}$.

El análisis para las Dimensiones Familiar e Individual, sin embargo, presentó disminuciones de sus valores promedio, antes y después del programa, estadísticamente significativas. Ambas dimensiones están extensamente estudiadas (Becoña, 2000; Muñoz-Rivas et al., 2001; Martínez et al., 2003) y sus resultados muestran principalmente como factores a considerar el apego fa-

12 Aunque se establecieron mecanismos para mejorar la confidencialidad de la información y esto a su vez se comunicó verbalmente a los estudiantes y se implementaron técnicas como el uso del cuestionario autoaplicado para aumentar la confianza y disminuir la abstención o la respuesta falaz. 
miliar, el uso de normas, supervisión parental (Lamborn et al., 1991; Stice et al., 1995; Jackson et al., 1997; Goselin et al., 2000; Di Clemente et al., 2001; citados en Martínez et al., 2003) y la actitud de los padres frente a las drogas (Muñoz-Rivas et al., 2001). El factor actitudinal del modelado parental, que corresponde a la posición que adoptan los padres y la familia en general hacia el consumo de drogas, son percibidas por los jóvenes como de igual o mayor importancia que el uso parental real (Pons et al., 1996, en Muñoz-Rivas et al., 2001).

Finalmente, se sostiene que contar con información respecto de la efectividad de las intervenciones sobre prevención de factores de riesgo, reduce no sólo los daños individuales y sociales vinculados con el consumo de drogas, sino que también abre la discusión a la responsabilidad de redefinir, adoptar nuevas estrategias, metodologías y teorías que apoyen decisiones con más pertinencia y mejor uso de los recursos. Y aunque este tipo de estudios toman una muestra reducida y específica del total, son relevantes justamente porque evalúan resultados particulares, en la perspectiva de validar experiencias y replicarlas a mayor escala.

\section{Bibliografía}

Alvira, F. (1999). Manual para la elaboración y evaluación de Programas de Prevención del Abuso de Drogas. España: Agencia Antidroga de la comunidad de Madrid.

ASH (2014). Young people and smoking fact sheet. Action on Smoking and Health. Recuperado de: http://ash. org.uk/files/documents/ASH_108.pdf

Becoña, E. (2000). Los Adolescentes y el Consumo de Drogas. Papeles del Psicólogo, nº 077. Consejo General de Colegios Oficiales de Psicólogos, pp. 25-32.

(2002). Bases científicas de la prevención de las drogodependencias. Delegación del Gobierno para el Plan Nacional sobre Drogas. España: Ministerio del Interior.

(2007). Bases psicológicas de la prevención del consumo de drogas. Papeles del Psicólogo, vol. 28, pp. 11-20.

Botvin, G.J. (2000). Preventing Adolescent Drug Abuse through Life Skills Training: Theory, Evidence of Effectiveness, and Implementation Issues. pp. 39-50. Cornell University. 
(2001). Life Skills Training Program. Princeton Health Press.

CSAP (1997). Guidelines and Benchmarks for Prevention Programing, National Center for the Advancement of Prevention.

Cohen, E. E R. Franco (2000). Evaluación de proyectos sociales. México: Siglo XXI.

Donaldson, L.J. (2009). Guidance on the Consumption of Alcohol by Children and Young People, From Sir Liam Donaldson, Chief Medical Officer for England Crown.

Espada, J.P.; Alcázar, A. E F.X. Méndez (2003). Eficacia de los Programas de Prevención Escolar de Drogas con Metodología Interactiva. Salud y Drogas, vol. 3, $n^{\circ}$ 2, pp. 61-81. España: Instituto de Investigación en Drogodependencia.

Faggiano, F.; Vigna-Taglianti, F.D.; Versino, E.; Zambon, A.; Borraccino, A. E P. Lemma (2007). School based prevention for ilicit drug use. A systematic review. Preventive Medicine, vol. 46, pp. 385-396.

Grant, B.F.; Stinson, F.S. E T.C. Harford (2001). Age of onset of alcohol use and DSM-IV alcohol abuse and dependence: A 12 year follow-up. Journal of Substance Abuse, 13, pp. 493-504.

Gottfredson, D.C. E Wilson, D.B. (2003). Characteristics of Effective School-Based Substance Abuse Prevention. Prevention Science, vol. $4 n^{\circ} 1$, pp. 27-38.

Hurtado, P. (2005). Consumo de drogas en detenidos: Aplicación de la metodología I- ADAM en Chile. Santiago de Chile: Fundación Paz Ciudadana. Recuperado de:http://psicologia.uahurtado.cl/seguridadurbana/docs/Consumo\%20de\%20drogas\%20 en\%20detenidos.pdf

Instituto Nacional para la Evaluación de la Educación, INEE (2010). El derecho a la educación en México. Informe 2009. México, DF.

James, C. (2011). Drug prevention programmes in schools: What is the evidence? Mentor. The Drug and Alcohol Protection Charity. UK. Recuperado de:http://www.mentoruk.org.uk/wp-content/ uploads/2011/11/Prevention-Evidence-PaperNov-11-Final.pdf 
Jit, M.; Barton, P.; Chen, Y.; Uthman, O.; Aveyard, P. \& C. Meads (2009). School-based interventions to prevent the uptake of smoking among children and young people: cost-effectiveness model. Recuperado de:http://www.nice.org.uk/guidance/ph23/ documents/schoolbased-interventions-toprevent-smoking-economic-modelling-reportfull-report2

Martinez, J.L.; Fuertes, A.; Ramos, M.; Hernández, A. (2003). Consumo de drogas en la adolescencia: importancia del afecto y la supervisión parental. Psicothema, vol. 15, n², pp. 161-166.

Miller, W. E S. Rollnick (1999). Entrevista motivacional. Preparar para el cambio de conductas adictivas. Barcelona: Editorial Paidós.

(2002). Motivational Interviewing. Preparing People for Change. Second Edition. New York: The Guilford Press.

Monreal, M.V.; Peroni, A.; Morris, P.; Jalón, R. Ed. (2009). Programas de Prevención del Consumo de Drogas, Programa de Tratamiento y Rehabilitación, Programa Previene (Municipios). Recuperado de: http://www.dipres.gob.cl/574/articles-49641_ doc_pdf.pdf

Muñoz-Rivas, Marina $\&$ José Luis Graña (2001). Factores familiares de riesgo y de protección para el consumo de drogas en adolescentes. Psicothema, vol. 13, $\mathrm{n}^{\circ} 1$, pp. 87-94.

NIDA (2006). Principles of Drug Abuse Treatment for Criminal Justice Populations.

OMS Organización Mundial de la Salud (2000). Clasificación de Trastornos Mentales CIE-10. Oficina de Publicaciones.

(2001). Informe Sobre la Salud en el Mundo. Salud mental: nuevos conocimientos, nuevas esperanzas. Oficina de Publicaciones.

OPS/OMS (2011). Agenda Estratégica. Cooperación técnica de la OPS/OMS con Chile 2011-2014. Recuperado de http://www.ocai.cl/opsoms20112014.pdf

Ortiz, E.; Sepúlveda, R. E Viano, C. (2005). Análisis de los programas de prevención dirigidos a jóvenes infractores de ley. Santiago de Chile: Universidad de Chile, Instituto de Asuntos Públicos. 
Peroni,A.ED. Keim, Comp. (2012). Investigación EvaluativadePolíticas Públicas, Programas y/o Proyectos Sociales. Relac Chile, Universidad de Chile. Recuperado de: http://evaluacionpoliticaspublicas.com/wpcontent/uploads/2014/09/Ponencias-Simposioisbn.pdf

Petterson, P.L.; Hawkins, J.D. E R.F. Catalano (1992). Evaluating comprensive community drug risk reduction interventions. Recuperado de: http://erx. sagepub.com/content/16/6/579.abstract

SENDA (2011). Análisis de Resultados Alcohol Use Disorders Identification Test (AUDIT). Noveno Estudio Nacional de Drogas en Población General de Chile. Boletín $\mathrm{N}^{\circ} 3$, Observatorio Chileno de Drogas. Santiago de Chile: Ministerio del Interior y Seguridad Pública.

(2012 a). A Tiempo. Una estrategia de detección precoz y trabajo preventivo del consumo de drogas y alcohol en adolescentes. Santiago de Chile: Ministerio del Interior y Seguridad Pública.

(2012 b). Escalas para uso problemático de sustancias. Recuperado de: http://www.cicad.oas.org/apps/ Document.aspx? $\mathrm{Id}=1289$

(2013). Estudios Nacionales de Drogas en Población Escolar en Chile. Recuperado de: http://www. senda.gob.cl/wp-content/uploads/2013/10/ InformeEjecutivoENPG2012.pdf

Trudel, M. E Puentes-Neuman, G. (2000). The contemporary concepts of at risk children: Theoretical models and approaches in the early 135 years. En Children ND Youth At Risk Symposium. Otawa, Canadá. Recuperado de: http://www.cesc-csce.ca/ pceradocs/2000/00Trudel_Puentes-Neuman_e. pdf

UNODC (2012). World Drug Report. United Nations publication, New York: NU. Recuperado de: http://www.unodc. org/documents/data-and-analysis/WDR2012/ WDR_2012_web_small.pdf

Villatoro, J.; Medina-Moro, M.E.; Rojano, C.; Fleiz, C.; Bermúdez, P.; Castro, P. E Juárez, F. (2002). ¿Ha cambiado el consumo de drogas de los estudiantes? Salud Mental, vol. 25, pp. 43-54. México: Instituto 
Evaluación de impactos de una intervención preventiva de drogras y alcohol / Carolina Núñez; José M. Merino

Nacional de Psiquiatría Ramón de la Fuente Muñiz.

Zweben, A. \& A. Zuckoff (2002). Motivational Interviewing and Treatment Adherence. Cognitive Behaviour Therapy, vol. 37, pp. 38-49. 